\title{
Spontaneous healing in maxillary fungus ball: Beware of asymptomatic patients
}

\author{
Antonio M. Bulfamante iD ｜ Carlotta Pipolo | Umberto D'Agostino Fiorenza | \\ Flavio Arnone | Paolo Lozza | Giovanni Felisati | Alberto Maria Saibene (iD
}

Otolaryngology Unit, Department of Health Sciences, Santi Paolo e Carlo Hospital, Università degli Studi di Milano, Milan, Italy

Correspondence

Alberto Maria Saibene, Otolaryngology Unit, ASST Santi Paolo e Carlo, Via Antonio di Rudinì, 8, 20142 Milan, Italy. Email: alberto.saibene@gmail.com

\begin{abstract}
Incidentally diagnosed, asymptomatic, paranasal fungus ball might occasionally undergo spontaneous healing. Accurate preoperative workup may avoid unnecessary surgical or anesthesiological procedures.
\end{abstract}

\section{K E Y W O R D S}

computed tomography, dental disease, endoscopy, maxillary sinus, paranasal sinus, rhinosinusitis

\section{1 | INTRODUCTION}

Paranasal sinus fungus balls (PSFB) are a noninvasive mycosis $^{1}$ which most commonly affects women in the 5 th and 6th decade of life. ${ }^{2}$ While their most frequent presentation is in the maxillary sinus, they can affect as well the other paranasal sinuses, ${ }^{2}$ sometimes even bilaterally. ${ }^{3}$

PSFB are a common sinonasal condition, and they underwent an unexplained incidence increase in the last decade. ${ }^{4}$ Some authors linked such an increase to the more widespread use of head imaging techniques (computed tomography and magnetic resonance) which allowed discovering a significant number of asymptomatic PSFB. ${ }^{5}$ While most fungus ball present with rhinosinusitis-like symptoms, ${ }^{6}$ most probably due to the frequent bacterial superinfection that characterizes these hyphal concretions, ${ }^{6,7}$ fungus ball might also be completely asymptomatic. ${ }^{8}$. Furthermore, PSFB have an unclear relationship with odontogenic sources of infection, ${ }^{9}$ and many papers associate endodontic material dispersion in the maxillary sinus as a possible facilitator of PSFB development. ${ }^{5,10}$

We present the case of a 78-year-old woman who was incidentally diagnosed with an odontogenic right maxillary sinus fungus ball who completely and spontaneously healed over the course of 1 year.

\section{2 | CASE PRESENTATION}

In October 2019, a 79-year-old female came to our otolaryngology outpatient service at the Santi Paolo e Carlo Hospital (Milan, Italy) reporting an incidental finding of a right maxillary, frontal, and ethmoidal sinus opacity at a recent head CT scan. The CT scan was prescribed by the patient's neurologist as a first-line evaluation for a holocranial headache. The CT scan images confirmed the right fronto-ethmoidmaxillary opacification and also showed a partial dental implant penetration in the maxillary sinus (see Figure 1), and hyperdense floccular bodies floating amid the isodense opacity (see Figure 2). The patient did not report any sinonasal symptoms (nasal breathing difficulties, hyposmia, sinus pressure, or rhinorrhea). Her history included only hypertension and type 2 diabetes mellitus, both correctly under treatment. The physical examination and routine blood tests were noncontributory. The oral examination did not show any sign of dental conditions with an adequate overall hygiene, and the patient, who had undergone implant dentistry 3 years before, was under regular dental follow-up. During ENT evaluation, nasal endoscopy showed a congested middle osteomeatal complex, with a significant uncinate process medialization. Based on clinical and radiological data, a diagnosis of

This is an open access article under the terms of the Creative Commons Attribution-NonCommercial-NoDerivs License, which permits use and distribution in any medium, provided the original work is properly cited, the use is non-commercial and no modifications or adaptations are made.

(C) 2020 The Authors. Clinical Case Reports published by John Wiley \& Sons Ltd. 


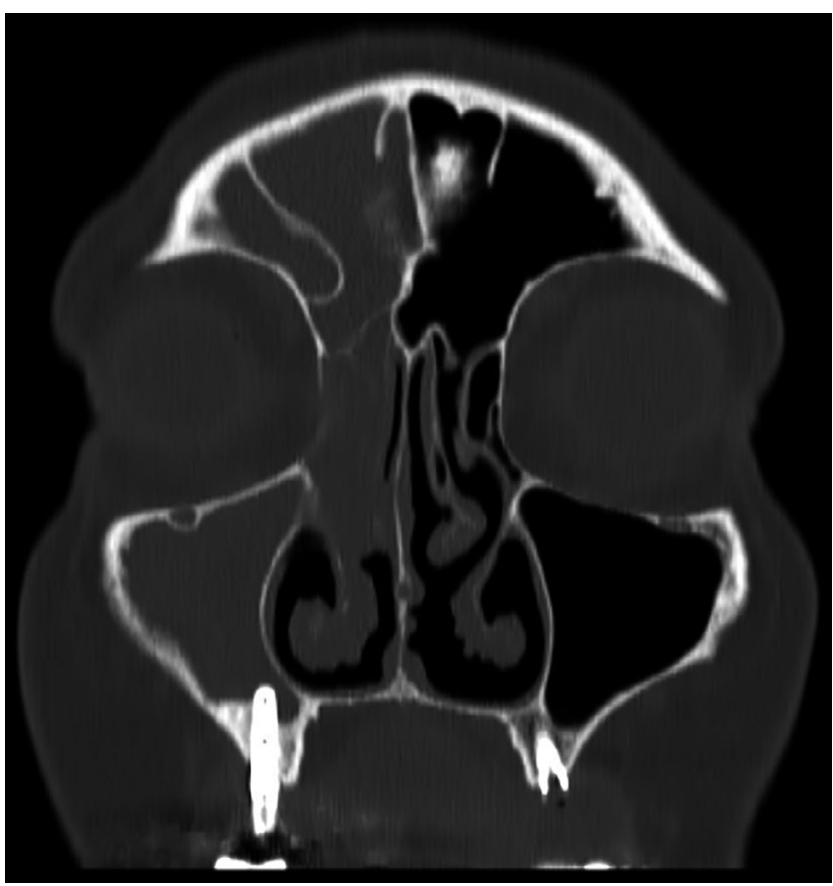

F I G U RE 1 Coronal head CT scan image showing a right fronto-ethmoid-maxillary sinus opacification, with a dental implant protruding into the right maxillary sinus

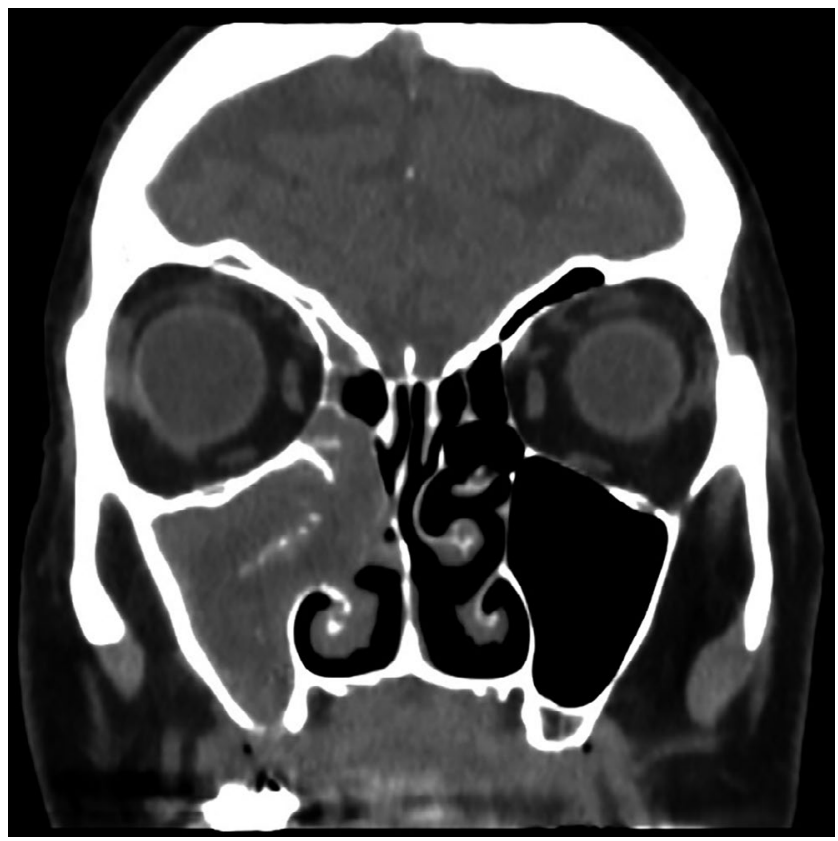

F I G U RE 2 Coronal head CT scan image showing hyperdense floccular bodies floating amid the isodense opacity, suggesting the diagnosis of a paranasal sinus fungus ball

PSFB was made, with an indication of surgical treatment. A functional endoscopic sinus surgery (FESS) procedure was planned, to be followed by dental evaluation for the protruding dental implant removal. Furthermore, a head MRI without contrast was prescribed to the patient prior to the surgical procedure, both to confirm the diagnosis of PSFB and to definitely rule out neoplastic processes given the unilaterality of the condition. Due to the internal reorganization of our ward and hospital following the Italian outbreak of SARSCoV-2, ${ }^{11}$ the patient could not perform the MRI until late September 2020, and surgery was postponed accordingly. No therapy was indicated meanwhile, being the patient completely free from symptoms. The MRI showed a complete resolution of the inflammatory process, without any sign of sinonasal opacification. Moreover, a wide opening of the right maxillary ostium was clearly visible (see Figures 3 and 4). A new flexible fiberoptic nasal endoscopy was performed immediately after the MRI. The endoscopy (see Video S1) confirmed the presence of a wide communication between the right nasal fossa and the ipsilateral maxillary sinus (see Figure 5), which appeared free from disease. The wide opening allowed a complete sinus exploration, which showed only some granulomatous tissue on the maxillary sinus floor, in adherence with the protruding dental implant (see Figure 6). On the left side, no sign of pathology was to be found. In the wake of these findings, we retracted the indication to FESS and the patient was sent for dental evaluation as had already been programmed. The dentist suggested the removal of the implant to avoid potential sinusitis recurrences. Nevertheless, the patient decided to leave the implant untouched and opted for a simple otolaryngologic follow-up. At present, the patient remains asymptomatic and no signs of recurring sinusitis or fungus ball have been reported.

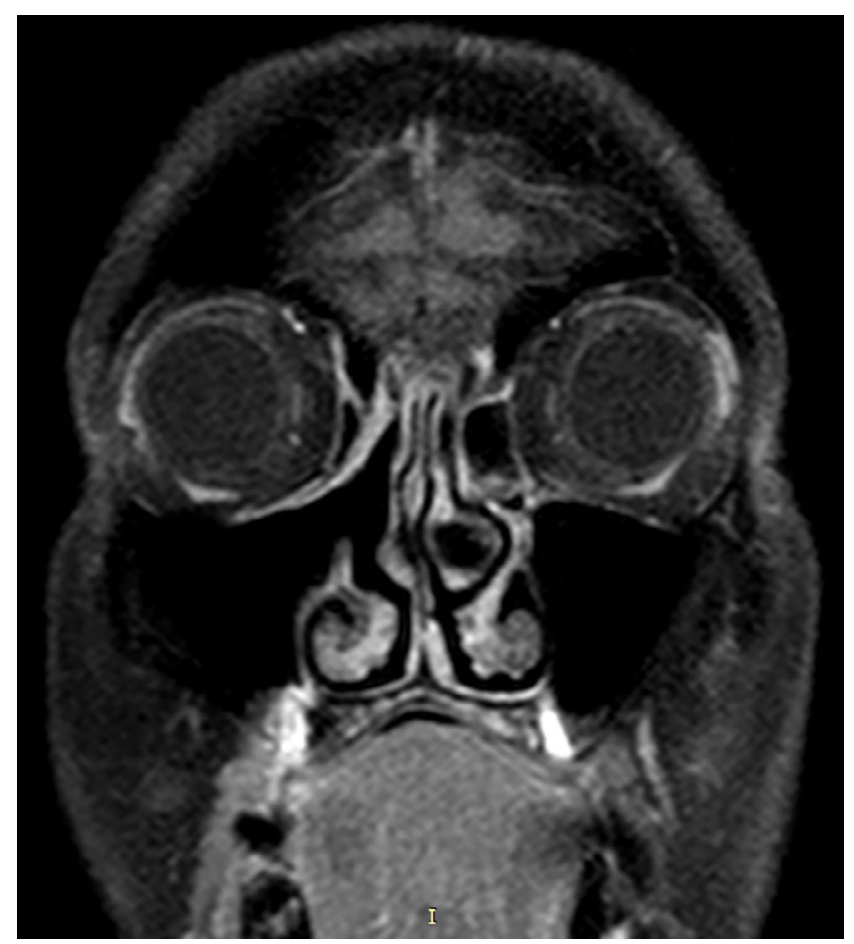

F I G U RE 3 T1-weighted coronal head MR image showing complete healing of the right maxillary sinus, with a wide antrostomylike aperture allowing for ventilation of the sinus 


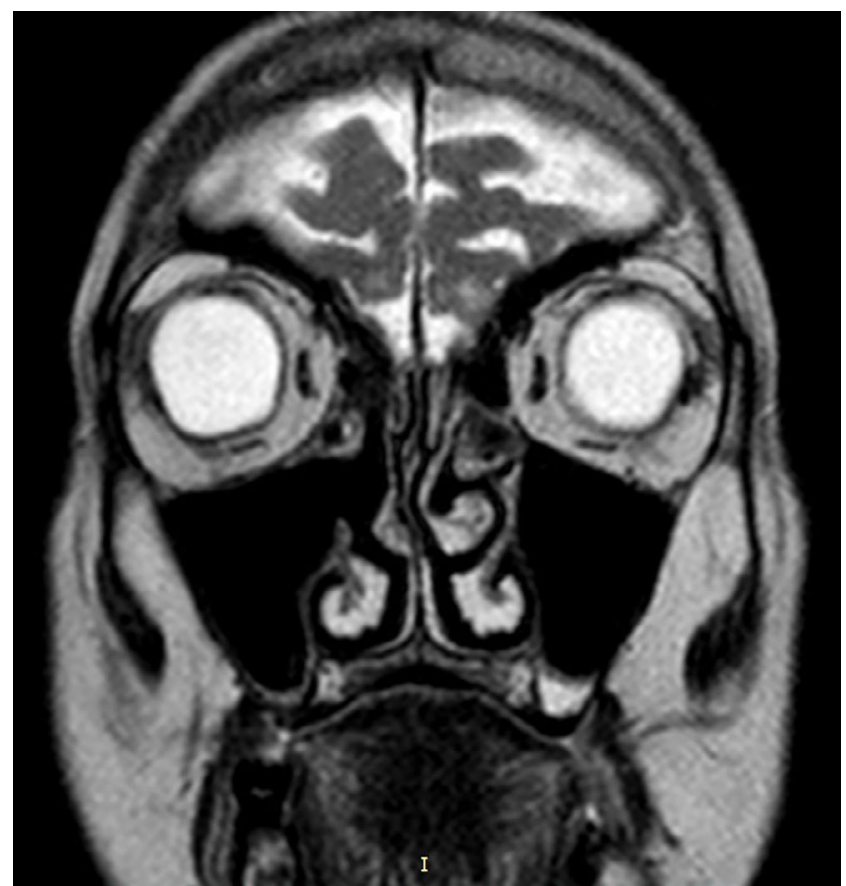

F I G U R E 4 T2-weighted coronal head MR image showing complete healing of the right maxillary sinus, with a wide antrostomylike aperture allowing for ventilation of the sinus

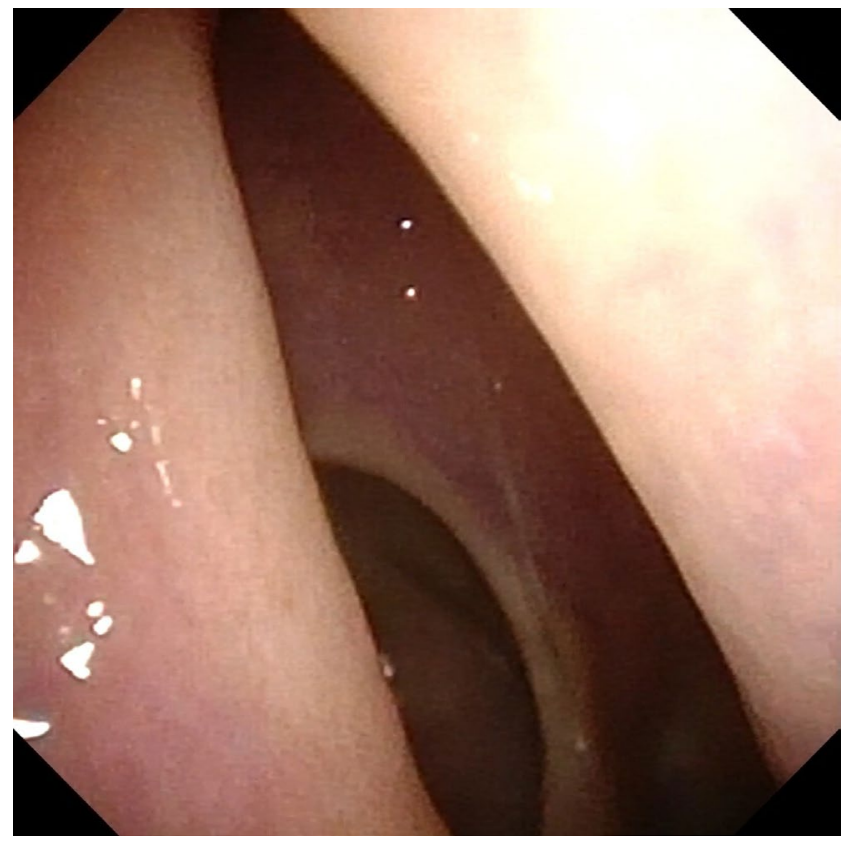

F I G U R E 5 Flexible nasal endoscopy image of the right middle meatus showing a complete opening of the right maxillary sinus, without any residual sign of paranasal sinus fungus ball

\section{DISCUSSION}

While uncommon, spontaneous healing of odontogenic sinonasal pathology has been documented over several publications, mostly related to small-sized oro-antral

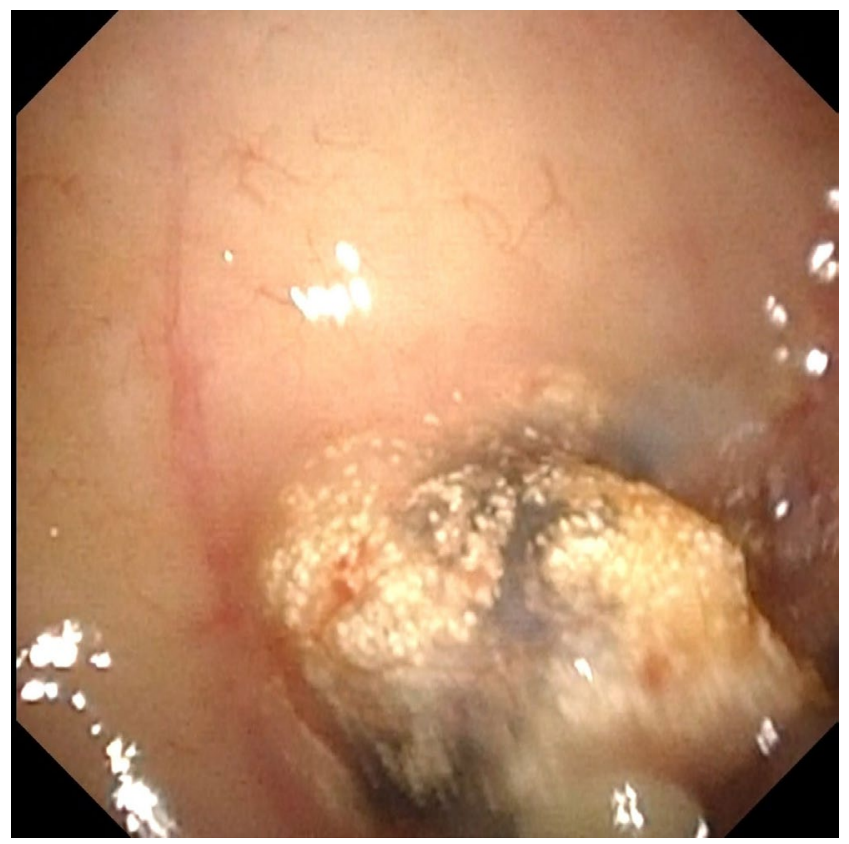

F I G U RE 6 Flexible nasal endoscopy image of the right maxillary sinus floor, showing a small amount of granulomatous tissue in adherence with the protruding dental implant

communications. ${ }^{12,13}$ Furthermore, if we focus again on odontogenic sinusitis, a number of authors propose treating the underlying dental condition as a possible means of achieving complete sinonasal healing, ${ }^{14}$ albeit current consensuses recommend such conservative approaches only for the treatment of asymptomatic or paucisymptomatic patients. ${ }^{15}$

Spontaneous healing of PSFB has not been described to date in the scientific literature, though the findings that characterize our case appear consistent with the patient history. The pathophysiologic mechanisms underlying this case might lie in the role of the uncinate process. As already described by Dal Pozzo et al, ${ }^{16}$ the uncinate process shows a significant medialization in odontogenic sinusitis patients, as in this specific case. Our hypothesis is that in this case the uncinate process, after extreme medialization under the push of the endosinusal content, might have acted as a failsafe valve. This mechanism, together with the mucociliary clearance of the sinus, might have allowed the hyphal and purulent content pressing on the maxillary sinus to leak from the sinus, preventing empyema, similarly to what happens in cystic fibrosis patients. ${ }^{17}$ In fact, mucociliary clearance as the primary innate defense mechanism might be the reason for the complete resolution of the disease after the opening of the medial wall of the sinus itself. Interestingly, the MCC is dependent on a multitude of external and internal factors such as $\mathrm{pH}$ changes, cigarette smoke, etc and the wave generated by the ciliated cells travels across the epithelium propelling the overlying mucus in a cephalad direction or toward the rhinopharynx. ${ }^{18}$ The ensuing sinus ventilation, altering the microenvironment and therefore the $\mathrm{pH}$, might have also 
allowed healing for a more efficient $\mathrm{MCC}$ and elimination of the PSFB. Of course, these hypotheses would require further validation but pose an intriguing clinical and scientific question. It has to be noted though that this patient's CT scan, despite being strongly suggestive for PSFB, cannot be considered diagnostically conclusive. The prescribed MRI might have helped the differential diagnosis between PSFB (showing area of signal void in T2-weighted sequences), a partially organized sinus empyema (isointense mucosal thickening and hypointense fluid in T1-weighted sequences and hyperintense mucosal thickening and endosinusal fluid in T2-weighted sequences), or a neoplastic process (with MRI characteristics related to histology). While patient's history allowed us to rule out a neoplastic process, we cannot exclude a priori that the fluid collection was due to an empyema. Nevertheless, the spontaneous resolution of such empyema through a spontaneously generated antrostomy still represents an interesting event, which does not change the overall teaching message of this case report.

Furthermore, the clinical course of this patient introduces two relevant data in the PSFB scenario. First and foremost, spontaneous healing following ventilation of the sinus seems to validate that PSFB, due to their noninvasive nature, can colonize the sinuses only given that specific microenvironment requirements are met (tendency to anaerobiosis in immune-privileged sites). ${ }^{7}$ Secondly, it might appear as if the relationship between PSFB and dental pathology lies simply in the facilitation of the fungal infection, whereas the simple dental focus persistence is not able to sustain the whole fungal infection. Conversely, it would be interesting to explore the relationship between fungal ball and prosthetic material infections, a relationship that has been thoroughly explored for bacterial biofilms. ${ }^{19}$ This specific case seems to suggest that fungus balls are not able to colonize implants, which remain free from infection after the fungus ball has been evacuated.

Another important point stemming from this report, which opens up a further set of clinical matters, is the clinical opportunity of surgery for asymptomatic PSFB. Was not for the pandemic contingency, this patient would have had an MRI confirming the PSFB and would have undergone surgery in a few weeks. Only the momentary stop in clinical and surgical activities allowed the scenario to evolve into a spontaneous healing which we would have never witnessed. Whether surgery for asymptomatic PSFB is unavoidable is a first question that emerges almost spontaneously. Further, it would be extremely interesting to explore the rate of asymptomatic PSFB that might actually evolve into spontaneous healing, a rate that only further prospective longitudinal studies could assess.

Besides these interesting pathophysiological speculations, this case report highlights without any doubt or inference of sorts how a complete preoperative workup of the patient is mandatory to avoid unnecessary treatments or surgeries. ${ }^{20}$
In the setting of an asymptomatic patient, nasal endoscopy and a careful evaluation of its findings represent an unsurpassed, noninvasive, and informative tool in the hands of otolaryngologists.

\section{CONFLICT OF INTEREST}

None declared.

\section{AUTHOR CONTRIBUTIONS}

AM Bulfamante and U D'Agostino Fiorenza: Writing and finalization of the article. Arnone F and Pipolo C: Patient evaluation and data collection. Lozza P and Felisati G: Paper revision. Saibene AM: Endoscopic evaluation, images, and drafting of the manuscript.

\section{ETHICAL APPROVAL}

We received informed consent from the patient for all the procedures described in this case report. The procedures performed do not deviate from the standard clinical protocols of care and required no further authorization. We conducted all the research pertaining to this article in accordance with the Helsinki Declaration.

\section{DATA AVAILABILITY STATEMENT}

Data pertaining to this article are available from the corresponding author upon reasonable request.

\section{ORCID}

Antonio M. Bulfamante (iD https://orcid. org/0000-0003-1812-6114

Alberto Maria Saibene (iD https://orcid. org/0000-0003-1457-6871

\section{REFERENCES}

1. deShazo RD, O'Brien M, Chapin K, Soto-Aguilar M, Gardner L, Swain R. A new classification and diagnostic criteria for invasive fungal sinusitis. Arch Otolaryngol Head Neck Surg. 1997;123:1181-1188.

2. Kim DW, Kim YM, Min J-Y, et al. Clinicopathologic characteristics of paranasal sinus fungus ball: retrospective, multicenter study in Korea. Eur Arch Otorhinolaryngol. 2020;277:761-765.

3. Vinciguerra A, Saibene AM, Lozza P, Maccari A. Unusual case of bilateral maxillary fungus ball. BMJ Case Rep. 2016;2016:115.https://doi.org/10.1136/bcr-2016-217930

4. Kim JS, So SS, Kwon SH. The increasing incidence of paranasal sinus fungus ball: a retrospective cohort study in two hundred forty-five patients for fifteen years. Clin Otolaryngol. 2017;42:175179. https://doi.org/10.1111/coa.12588

5. Costa F, Emanuelli E, Franz L, Tel A, Sembronio S, Robiony M. Fungus ball of the maxillary sinus: retrospective study of 48 patients and review of the literature. Am J Otolaryngol. 2019;40:700704. https://doi.org/10.1016/j.amjoto.2019.06.006

6. Liu X, Liu C, Wei H, et al. A retrospective analysis of $1,717 \mathrm{pa}-$ ranasal sinus fungus ball cases from 2008 to 2017. Laryngoscope. 2020;130:75-79. 
7. Saibene AM, Vassena C, Pipolo C, et al. Odontogenic and rhinogenic chronic sinusitis: a modern microbiological comparison. Int Forum Allergy Rhinol. 2016;6:41-45.

8. Park W-B, Kim Y-J, Park JS, Han J-Y, Lim H-C. Complication and salvage of sinus floor elevation in the maxillary sinus with asymptomatic and non-calcified fungus colonization: a case report. J Oral Implantol. 2020. https://doi.org/10.1563/ aaid-joi-D-20-00127

9. Molteni M, Bulfamante AM, Pipolo C, et al. Odontogenic sinusitis and sinonasal complications of dental treatments: a retrospective case series of 480 patients with critical assessment of the current classification. Acta Otorhinolaryngol Ital. 2020;40:282-289.

10. Nicolai P, Mensi M, Marsili F, et al. Maxillary fungus ball: zinc-oxide endodontic materials as a risk factor. Acta Otorhinolaryngol Ital. 2015;35:93-96.

11. Saibene AM, Allevi F, Biglioli F, Felisati G. Role and management of a head and neck department during the COVID19 outbreak in Lombardy. Otolaryngol Head Neck Surg. 2020;162:795-796.

12. Khandelwal P, Hajira N. Management of oro-antral communication and fistula: various surgical options. World J Plast Surg. 2017;6:3-8.

13. Scarano A, Piattelli A, Iezzi G, Varvara G. Spontaneous bone formation on the maxillary sinus floor in association with surgery to remove a migrated dental implant: a case report. Minerva Stomatol. 2014;63:351-359.

14. Akiyama K, Nakai Y, Samukawa Y, Miyake M, Hoshikawa H. Assessment of simultaneous surgery for odontogenic sinusitis: endoscopic sinus surgery with endoscopic apicoectomy. J Craniofac Surg. 2019;30:239-243.

15. Craig JR, Tataryn RW, Aghaloo TL, et al. Management of odontogenic sinusitis: multidisciplinary consensus statement. Int Forum Allergy Rhinol. 2020;10:901-912.

16. Uncinate process deviation in patients with odontogenic sinusitis: a computed tomographic evaluation. [cited 30 Oct 2020]. https:// search.proquest.com/openview/46ce919d307e88604dcca43c5 $680 \mathrm{e} 3 \mathrm{ec} / 1$ ?pq-origsite $=$ gscholar $\& \mathrm{cbl}=756425$. Accessed October 28, 2020.

17. Kim HJ, Friedman EM, Sulek M, Duncan NO, McCluggage C. Paranasal sinus development in chronic sinusitis, cystic fibrosis, and normal comparison population: a computerized tomography correlation study. Am J Rhinol. 1997;11:275-281.

18. Bustamante-Marin XM, Ostrowski LE. Cilia and Mucociliary Clearance. Cold Spring Harbor Perspectives in Biology. 2017;9:a028241. https://doi.org/10.1101/cshperspect.a028241

19. Sridhar S, Wang F, Wilson TG, Palmer K, Valderrama P, Rodrigues DC. The role of bacterial biofilm and mechanical forces in modulating dental implant failures. J Mech Behav Biomed Mater. 2019;92:118-127.

20. Lal D, Rounds A, Dodick DW. Comprehensive management of patients presenting to the otolaryngologist for Sinus pressure, pain, or headache. The Laryngoscope. 2015;125:303-310. https://doi. org/10.1002/lary.24926

\section{SUPPORTING INFORMATION}

Additional supporting information may be found online in the Supporting Information section.

How to cite this article: Bulfamante AM, Pipolo C, D'Agostino Fiorenza U, et al. Spontaneous healing in maxillary fungus ball: Beware of asymptomatic patients. Clin Case Rep. 2020;00:1-5. https://doi. org/10.1002/ccr3.3665 